\title{
Estado y políticas públicas desde una perspectiva crítica
}

\author{
State and public policies from a critical perspective \\ Estado e políticas públicas sob uma perspectiva crítica
}

Recebido: 07 abr 2019

Revisado: 08 abr 2019

Aceito: 18 mar 2020

\section{Autor de}

correspondência:

Alberto Bonnet

abonnetprivado@gmail.com

Conflito de interesses: Os autores declaram não haver nenhum interesse profissional ou pessoal que possa gerar conflito de interesses em relação a este manuscrito.

Este artículo se basa en la conferencia "Análise crítica das políticas públicas no capitalismo contemporâneo", dictada por Alberto Bonnet en el marco del I Seminario Internacional de Pesquisa em Economia Política da Saúde" el 15 de febrero de 2019 en la Faculdade de Saúde Pública de la Universidade de São Paulo.

\section{Resumen}

Este artículo parte de la teoría crítica del estado capitalista y, más específicamente, del denominado "debate sobre derivación del estado", para esbozar a continuación algunas características que revestiría un análisis crítico del proceso de las políticas públicas. A diferencia de la teoría del estado, el análisis de las políticas públicas es una sub-disciplina que carece de perspectivas críticas. La intención de este artículo es revisar la relación existente entre las políticas públicas y los requerimientos de la reproducción capitalista y, a partir de esta revisión, proponer algunos elementos para una concepción del proceso de políticas públicas en términos de un proceso de ensayo y error.

Palabras-claves: Estado; Políticas Públicas; Capitalismo.

\section{Abstract}

This article starts from the critical theory of the capitalist state and, more specifically, from the socalled "state-derivation debate", to outline some characteristics that would have a critical analysis of the public policy process. Unlike state theory, the analysis of public policies is a sub-discipline that lacks critical perspectives. The intention of this article is to review the relationship between public policies and the requirements of capitalist reproduction and, from this review, propose some elements for a conception of the political policy process in terms of a trial and error process.

Keywords: State; Public Policies; Capitalism.

\section{Resumo}

Este artigo parte da teoria crítica do estado capitalista e, mais especificamente, do chamado "debate sobre a derivação do estado", para delinear algumas características que teriam uma análise crítica do processo de políticas públicas. Diferentemente da teoria do estado, a análise de políticas públicas é uma sub-disciplina que carece de perspectivas críticas. A intenção deste artigo é revisar a relação entre políticas públicas e os requisitos de reprodução capitalista e, a partir dessa revisão, propor alguns elementos para uma concepção do processo de política pública em termos de processo de tentativa e erro.

Descritores: Estado; Políticas Públicas; Capitalismo. 


\section{Introducción}

El ciclo de auge de los conflictos sociales y de crisis del neoliberalismo registrado entre fines de la década de los noventa y comienzos de la siguiente en varios países latinoamericanos - incluido Brasil - permitieron el ascenso al poder de una serie de gobiernos de orientación progresista o populista. En este nuevo escenario se renovaron, entre amplios sectores de la sociedad en general y de la intelectualidad en particular, las esperanzas en la intervención del estado como recurso privilegiado para impulsar el desarrollo económico y la inclusión social en la región. Estas renovadas esperanzas en la intervención del estado volvieron a poner en agenda a su vez la problemática del estado capitalista y sus funciones, más específicamente, el alcance de las políticas públicas y su relación con la reproducción capitalista. Aquel ciclo de gobiernos progresistas o populistas en el poder parece haberse clausurado en nuestros días - de la manera más dramática en el caso de Brasil. Sin embargo, esta pregunta por el alcance de las políticas públicas y su relación con la reproducción capitalista conserva su vigencia.

La intención de este artículo es revisar esa relación entre las políticas públicas y la reproducción capitalista y, a partir de esa revisión, proponer elementos para una concepción crítica del proceso de políticas públicas en términos de un proceso de ensayo y error. Los límites que nos imponen estas pocas páginas nos obligan a esbozar apenas esos elementos, que hemos desarrollado más extensamente en otros trabajos previos. ${ }^{1,2}$ Pero creemos que, a pesar de estos límites, la ausencia de perspectivas críticas dentro de un campo del análisis de las políticas públicas que sigue dominado por el mainstream anglosajón justifica estas páginas en una revista interesada en las políticas de salud pública.

\section{Del estado a las políticas públicas}

Precisemos nuestro problema. El análisis de las políticas públicas es, por la propia naturaleza de su objeto, una dimensión de la teoría del estado. Y esto nos enfrenta a una aparente paradoja. Existe, por una parte, una extensa bibliografía de teoría crítica del estado moderno en tanto estado capitalista, proveniente principalmente de la tradición marxista. Pero no existen, por otra parte, sino muy escasos aportes específicos a un análisis crítico del proceso de las políticas públicas. Esta aparente paradoja encuentra su explicación en las características de dos concepciones que dominaron el desarrollo de la teoría marxista del estado durante décadas, que aquí vamos a denominar como las concepciones economicistadeterminista y politicista-instrumentalista del estado.

En efecto, aunque concebían la relación entre estado y capital de modos diferentes, ambas concepciones compartían la tendencia a presuponer un alto grado de adecuación, al 
menos en los momentos de la definición de los problemas y la construcción de las agendas, la formulación de las políticas y la decisión de implementarlas, entre las políticas públicas y los requerimientos de la reproducción capitalista. La economicista-determinista partía de una lectura mecánica de la metáfora marxiana de base / superestructura y concebía al estado como un mero apéndice del capital. Dentro de esta concepción, las presuntas necesidades del capital parecían imponerse por si mismas sobre las políticas públicas, de manera que la adecuación de estas políticas públicas a los requerimientos de la reproducción capitalista estaba garantizada de antemano y el análisis específico del proceso de las políticas públicas resultaba superfluo. La politicista-instrumentalista, en cambio, concebía al estado como un instrumento de la clase dominante. Esta concepción parecía poder rendir cuenta en alguna medida de los mecanismos que garantizaban la adecuación de las políticas públicas a los requerimientos de la reproducción capitalista mediante la investigación de los vínculos existentes entre el personal del estado y la clase dominante. Pero la reducción del estado a un simple instrumento de la burguesía volvía a convertir el análisis específico del proceso de esas políticas públicas en algo relativamente superfluo.

Este panorama se modificó definitivamente con la renovación que atravesó la teoría marxista del estado a fines de los sesenta y comienzos de los setenta. Y dentro de esta multifacética renovación teórica resultó especialmente relevante, en cuanto a su riqueza en sí mismo y para el problema que nos ocupa, el denominado debate sobre la derivación del estado (o Staatsableitungsdebatte) desarrollado en la ex República Federal de Alemania en la primera mitad de los setenta. ${ }^{3}$ El punto de partida compartido por los intervinientes en este debate fue la separación entre lo económico y lo político específica y constitutiva de la sociedad capitalista, separación que el joven Marx ya había heredado de Hegel y elaboraría en sus escritos posteriores. Esta separación entre lo económico y lo político implica que las relaciones sociales, en la sociedad capitalista, adoptan dos formas o modos de existencia fundamentales diferenciados: las formas capital y estado. Esta particularización del estado capitalista como forma diferenciada es condición de posibilidad de su propia existencia y a la vez le impone límites sistémicos estrictos, aunque cambiantes en sus manifestaciones históricas, a su capacidad de intervención en la sociedad. ${ }^{4}$ Los intervinientes en el debate estaban interesados especialmente, en su contexto, en identificar los límites que enfrentaban las políticas económicas anti-cíclicas del estado keynesiano de posguerra ante las crisis de acumulación. Pero el punto que nos interesa remarcar en este contexto nuestro es que algunos de los participantes, como Hirsch ${ }^{5}$ y Wirth, ${ }^{6}$ no sólo problematizaron ese alcance de la intervención del estado sino también su propia funcionalidad. La adecuación de las 
políticas públicas a los requerimientos de la reproducción capitalista se convertía así en un auténtico problema.

Esta problematización de la relación entre las políticas públicas y los requerimientos de la reproducción capitalista reclamaba, naturalmente, un análisis específico del proceso

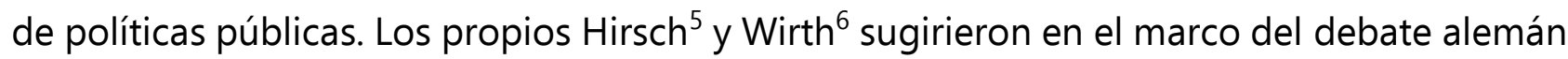
un camino para encarar dicho análisis mediante la hipótesis de que ese proceso de las políticas públicas no estaba ni podía estar regido por la racionalidad perfecta que le había atribuido el mainstream del análisis de políticas públicas, por lo menos en los orígenes de esta sub-disciplina, sino que estaba regido por una racionalidad adaptativa. El proceso de las políticas públicas, en este sentido, revestía más bien la forma de un proceso de ensayo y error. Algunos autores posteriores, desde Holloway ${ }^{7}$ y $\mathrm{O}^{\prime}$ Donnell ${ }^{8}$ en aquellos años hasta Brand $^{9}$ en nuestros días, recuperaron aquella hipótesis. Sin embargo, el desarrollo de un análisis crítico sistemático del proceso de las políticas públicas en esta clave sigue siendo una tarea pendiente.

Por cierto, dentro del propio mainstream del análisis de políticas públicas, aquella racionalidad perfecta fue puesta en entredicho muy temprano - en los trabajos pioneros de Simon ${ }^{10}$ y Lindblom ${ }^{11}$ de fines de los cincuenta - y se desarrolló un análisis heterodoxo del proceso de las políticas en términos de ensayo y error. Sin embargo, la concepción pluralista del estado y la sociedad y marginalista del mercado que enmarcaron este análisis del proceso de las políticas públicas impiden que pueda asimilarse sin más dentro del marco de una teoría crítica del estado. Veamos, en el siguiente apartado, algunas características que revestiría un análisis crítico del proceso de las políticas públicas como proceso de ensayo y error.

\section{Las políticas públicas como ensayo y error}

El rechazo simultáneo de las concepciones economicista-determinista y politicistainstrumentalista del estado, decíamos, convierte a la adecuación de las políticas públicas a los requerimientos de la reproducción capitalista en un problema. La hipótesis de que el proceso de políticas reviste la forma de un proceso de ensayo y error apunta precisamente a explicar esa relación de una manera rigurosamente objetiva, es decir, independiente de la influencia que la clase capitalista pueda ejercer sobre el comportamiento del personal del estado, pero que sea a la vez capaz de rendir cuenta del comportamiento de ese personal del estado y de las características del aparato de estado involucrados en el proceso de políticas públicas, es decir, de los mecanismos específicos a través de los cuales se establece aquella relación.

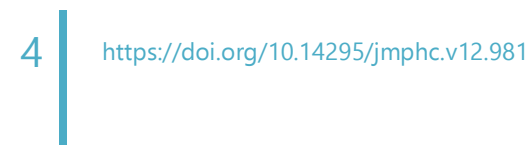


Para precisar las características que reviste esta relación y que determinan el hecho de que el proceso de las políticas públicas adopte esa forma de ensayo y error debemos recordar algunas características constitutivas de ambos polos de la relación, a saber, del estado y del capital. La particularización del estado en tanto forma diferenciada de las relaciones sociales capitalistas, que mencionamos antes, se materializa en la conformación de un aparato de estado diferenciado de las restantes instancias organizativas de la sociedad y de un personal político y burocrático de estado igualmente diferenciado de los restantes grupos sociales. Este personal de estado posee inevitablemente un interés específico en su propia auto-conservación, dependiente a su vez de la conservación de sus posiciones dentro del aparato de estado y de la reproducción de ese aparato de estado en sí mismo, interés que no coincide inmediatamente con los intereses de ninguna clase o fracción de clase existente en la sociedad. El personal de estado no es, en ningún caso, una mera correa de transmisión de los intereses de la burguesía.

La reproducción del capital, por su parte, es un proceso anárquico que se desarrolla, a espaldas de los propios agentes involucrados, a través de la lucha de clases (derivada del antagonismo entre capital y trabajo) y de los conflictos entre fracciones de la burguesía (derivados de la competencia). Naturalmente, no existe nada semejante a un interés general en una sociedad de clases. Puede postularse teóricamente, en cambio, que existe un interés colectivo del capital en su conjunto, pero este interés colectivo no es accesible para los capitalistas individuales ni para sus organizaciones. Los mencionados requerimientos de la reproducción capitalista son entonces, en gran medida, insondables tanto para los propios capitalistas como para los miembros del personal de estado.

Estado y capital, sin embargo, no guardan entre sí una relación de exterioridad. No son dos relaciones sociales diferentes, sino dos modos de existencia diferenciados de unas mismas relaciones sociales. Esto implica, por una parte, que la reproducción del estado es inseparable de la reproducción del capital. La reproducción del aparato y del personal del estado depende materialmente, en última instancia, de la reproducción ampliada del capital. E implica además que esas características que reviste la anárquica reproducción del capital se inscriben, aunque siempre de una manera específica, en la reproducción del estado. La reproducción del estado en la esfera pública descansa en mecanismos más centralizados y conscientes que la ciega reproducción del capital en la esfera privada. Pero esto no impide que aquella lucha de clases y aquellos conflictos inter-burgueses se inscriban en los propios aparato y personal del estado. En consecuencia, el estado no es una entidad monolítica, sino un agregado signado en mayor o menor medida por la fragmentariedad y la incoherencia. ${ }^{12}$ 
Agreguemos finalmente que el estado, en su constitución como estado nacional, no es co-extensivo con el capital. En efecto, mientras que el escenario de la reproducción del capital es por definición y cada vez en mayor medida el mercado mundial, el escenario del proceso de las políticas públicas sigue siendo en gran medida un escenario doméstico. No es necesario detenerse aquí en las limitaciones que esta restricción impone al alcance de estas políticas públicas. ${ }^{13}$

Estas son las condiciones estructurales en las que se desenvuelve el proceso de las políticas públicas en la sociedad capitalista. La medida y el modo en que estas condiciones se ponen de manifiesto varían, naturalmente, según el contexto histórico-geográfico del que se trate. Los intereses del personal de estado se diferencian escasamente de los intereses de la clase dominante en los orígenes de los estados capitalistas -en las formas oligárquicas que revistieron los estados latinoamericanos en sus comienzos, por ejemplo. La manera en que se manifiestan la fragmentariedad y la incoherencia que signan al aparato y al personal del estado depende de los regímenes políticos y de las coyunturas en cuestión -en las dictaduras militares latinoamericanas se manifestaban como pugnas entre camarillas, por ejemplo, mientras que en las democracias que las sucedieron se expresan a través del parlamento y de otras instituciones formales. Y así sucesivamente. Pero, en cualquier caso, esas condiciones no son coyunturales sino estructurales, pues derivan de las características que reviste la reproducción de la sociedad capitalista.

Estas condiciones estructurales determinan que el proceso de las políticas públicas adopte la forma de un proceso de ensayo y error. $Y$, en este sentido, puede afirmarse que el carácter específicamente capitalista que reviste ese proceso de las políticas públicas radica antes en esta forma que adopta que en sus variables contenidos. Pero describamos, en una versión muy estilizada, este proceso. Los requerimientos de la reproducción capitalista no se presentan ante el personal del estado como tales, sino como una diversidad de demandas diferentes, opuestas y a menudo contradictorias entre sí, planteadas por organizaciones que representan intereses de distintos grupos sociales: fracciones de la burguesía, sectores del proletariado, ocasionalmente grandes empresas individuales. Las vagas preferencias que la ciudadanía manifiesta en las elecciones, mientras tanto, juegan un papel completamente secundario. La propia naturaleza de estas demandas, innumerables, divergentes, imposibles de satisfacer dentro de las condiciones vigentes en muchos casos, exige del personal político del estado una tarea de selección y redefinición de demandas previo a su ingreso en agenda. En los hechos, muchas demandas no ingresan en agenda y muy pocas ingresan en sus versiones originales. El interés de este personal político en su auto-conservación incide decisivamente en los criterios a partir de los cuales realiza esta selección y redefinición. Los 
dirigentes del estado se comportan así de una manera activa, ciertamente, aunque normalmente reactiva.

Las demandas que ingresan en la agenda de las políticas públicas, por su parte, pueden permanecer indefinidamente en esa agenda. En los casos en que dan lugar a un proceso de formulación de políticas, el personal político del estado se ve enfrentado a una tarea de búsqueda de respuestas que, dada la estructura de la sociedad capitalista, resulta muy ardua porque esa estructura suele interponer un abismo insalvable entre las causas de los problemas que suscitan aquellas demandas y el modo en que se manifiestan en la superficie de la sociedad. Problemas tales como la inflación, la inseguridad o el racismo suelen aparecer en la superficie, tanto para quienes los convierten en demandas suyas en el seno de la sociedad como para quienes recogen esas demandas desde el estado, por así decirlo, como meros síntomas de innumerables enfermedades posibles. La formulación y la decisión sobre de políticas públicas no aspira normalmente en consecuencia a la resolución de problemas, en un sentido estricto, sino a su mera desaparición de la agenda. La implementación de esas políticas en manos del personal burocrático del estado, finalmente, acarrea siempre en los hechos algún grado de reformulación de las mismas.

Las políticas implementadas como resultado de este complejo proceso revisten en consecuencia el status de meros intentos. Si estos intentos son exitosos, es decir, si logran suprimir de la agenda las demandas que originaron esas políticas, el personal político del estado tiende inercialmente a insistir en el rumbo trazado por esas políticas. Si fracasan, en cambio, se ve forzado a modificar ese rumbo bajo la amenaza de verse desplazado de sus cargos. La orientación político-ideológica de este personal de estado suele subordinarse a estos mecanismos objetivos -y no es casual en este sentido, por ejemplo, que las reformas neoliberales hayan sido implementadas en varios países latinoamericanos por gobiernos provenientes de partidos populistas.

La relación entre las políticas públicas y los requerimientos de la reproducción capitalista se establece a través de este proceso de ensayo y error. Una adecuación completa de las políticas públicas a los requerimientos de la reproducción capitalista es inalcanzable porque estos requerimientos son en sí mismos contradictorios. Una completa inadecuación entre las políticas públicas y los requerimientos de la reproducción capitalista resulta a su vez insostenible porque desemboca en crisis de acumulación y dominación que fuerzan al personal de estado a modificarlas. ${ }^{14}$ El mecanismo de ensayo y error se convierte así en un mecanismo de adaptación y de aprendizaje para los dirigentes del estado. 


\section{Agradecimientos}

Agradeço imensamente ao Áquilas Mendes e ao Grupo de Pesquisa "Saúde, Estado e Capitalismo Contemporâneo" pelo convite e possibilidade de participar nesse "I Seminário Internacional de Pesquisa em Economia Política da Saúde" e compartilhar a mesa com a professora Ivanete Salete Boschetti.

\section{Referencias}

1. Alvarez Huwiler L, Bonnet A. Ensayo y error. Un análisis marxista de las políticas públicas. Rev Mex Cienc Polit Soc. 2018;63(233): 169-92. http://dx.doi.org/10.22201/fcpys.2448492xe.2018.233.59461.

2. Alvarez Huwiler $L$, Bonnet $A$. Aportes para una crítica marxista de las políticas públicas. In: Mallardi M, Fernández E, compiladores Cuestión social y políticas sociales. Crítica a sus fundamentos y expresiones contemporáneas. Mar del Plata: Universidad Nacional de Mar del Plata; 2019. p. 107-36.

3. Bonnet A, Piva A, editores. Estado y capital: el debate alemán sobre la derivación del estado. Buenos Aires: Herramienta; 2017. 724 p. Disponible en: https://herramienta.com.ar/sites/default/files/Estado_y_Capital.pdf.

4. Blanke $B$, Jürgens $U$, Kastendiek $H$. Acerca de la reciente discusión marxista sobre el análisis de la forma y función del estado burgués. Reflexiones sobre la relación entre política y economía. In: Bonnet A, Piva A, editores. Estado y capital: el debate alemán sobre la derivación del estado. Buenos Aires: Herramienta; 2017; p. 589-656. Disponible en: https://herramienta.com.ar/sites/default/files/Estado_y_Capital.pdf.

5. Hirsch J. Elementos para una teoría materialista del estado. In: Bonnet A, Piva A, editores. Estado y capital: el debate alemán sobre la derivación del estado. Buenos Aires: Herramienta; 2017.p. 441-508. Disponible en: https://herramienta.com.ar/sites/default/files/Estado_y_Capital.pdf.

6. Wirth M. Acerca de la crítica de la teoría del capital monopolista de estado. In: Bonnet A, Piva A. editores. Estado y capital: el debate alemán sobre la derivación del estado. Buenos Aires: Herramienta; 2017. p. 401-440. Disponible en: https://herramienta.com.ar/sites/default/files/Estado_y_Capital.pdf.

7. Holloway J. Fundamentos teóricos para una crítica marxista de la administración pública. México, DF: Instituto Nacional de Administración Pública; 1982. 92 p. 
8. O’Donnell G. Apuntes para una teoría del estado. In: Rev Mex Sociol.1978:40(4):1157-99. http://dx.doi.org/10.2307/3539655

9. Brand U. State, context and correspondence. Contours of a historical-materialist policy analysis. In Österreichische Zeitschrift für Politikwissenschaft. 2013;42(4):425-42. https://doi.org/10.15203/ozp.129.vol42iss4.

10. Simon H. Models of man: Essays in memory of Herbert A. Simon. New York: John Wiley and Sons; 1957. A behavioral model of rational choice; p. 26-30.

11. Lindblom CE. The science of 'muddling through'. Public Adm Rev. 1959;19(2):79-88. https://doi.org/10.2307/973677.

12. Poulantzas N. Estado, poder y socialismo. México: Siglo XXI; 1975. 326 p.

13. Holloway J. Global capital and the national state. Cap Class. 1994;18(1):23-49. https://doi.org/10.1177/030981689405200103

14. Block F. Revising state theory. Essays in politics and postindustrialism. Filadelfia: Temple University Press; 1987 . The ruling class does not rule: notes on the marxist theory of the state. p. 51-68.

\section{Minicurrículo}

\section{Alberto Bonnet}

Universidad Nacional de Quilmes - UNQ, Bernal, y Universidad de Buenos Aires - UBA, Buenos Aires, Argentina. Doctor en Ciencias Sociales, Instituto de Ciencias Sociales y Humanidades - Universidad Autónoma de Puebla, México.

Laura Alvarez Huwiler | ORCiD: 0000-0001-8170-8702

Universidad Nacional de Quilmes - UNQ, Bernal, Argentina. Doctora en Ciencias Sociales, Facultad de Ciencias Sociales - UBA y Pos-doctora en el Programa Trandes "Desarrollo Sostenible y Desigualdades Sociales en la Región Andina", Freie Universität Berlin y Pontificia Universidad Católica del Perú. 